BA-TH/99-363

September 1999

gr-qc/9910019

\title{
On the response of gravitational antennas to dilatonic waves
}

\author{
M. Gasperini \\ Dipartimento di Fisica, Università di Bari, \\ Via G. Amendola 173, 70126 Bari, Italy \\ and \\ Istituto Nazionale di Fisica Nucleare, Sezione di Bari, Bari, Italy
}

\begin{abstract}
It is pointed out that the coupling of macroscopic test masses to the gravidilaton background of string theory is non geodesic, in general, and cannot be parametrized by a Brans-Dicke model of scalar-tensor gravity. The response of gravitational antennas to dilatonic waves should be analyzed through a generalized equation of geodesic deviation, taking into account the possible direct coupling of the background to the (composition-dependent) dilatonic charge of the antenna.
\end{abstract}

Typeset using REVTEX 
A number of papers has recently explored the interesting possibility of detecting scalar waves, with coupling strength to matter of gravitational order, exploiting both resonant mass [1] - [3] and interferometric [4, [0] gravity wave detectors. Indeed, gravitational antennas conceived to respond to the tensor part of metric fluctuations can also respond to the scalar oscillations of the metric background, induced by the coupling to some fundamental scalar component of the gravitational multiplet.

Such papers are basically motivated by the possible emission of scalar waves in astrophysical process, like the spherically symmetric collapse of a star, or of a cloud of dust [2, 6]. Another motivation is the possible presence or a relic cosmic background of (light enough) scalar particles. Such particles could be produced copiously in the early Universe (see for instance [7,8]), and could survive until today in the form of a stochastic background of scalar waves, representing a possible significant fraction of the present large scale density of dark matter. Given the extreme weakness of their couplings, gravitational antennas are probably (at present) the only plausible candidates for their direct detection [9].

In all the quoted papers [1] - [5], the analysis of the possible response of the detector was performed assuming a geodesic coupling of the test masses (representing the detector) to the scalar component of the metric fluctuations. The standard equation of geodesic deviation was subsequently applied to estimate the detector sensitivity. This is certainly justified when the gravitational scalar-tensor interactions are parametrized by a strictly "Brans-Dicke type" model of gravity, as always assumed in [1] - [5]. In that case, a "Jordan frame" exists in which there are no couplings to the scalar field in the matter part of the action, and the scalar interactions are totally absorbed in the rescaled metric.

This is not the most general case, however, and one of the purposes of this paper is to point out that this is not the case, in particular, for the coupling of macroscopic test masses to the scalar dilaton field appearing in unified theories of strings and superstrings [10]. In that context, in fact, ordinary macroscopic masses have a scalar "dilatonic charge", which is in general non-universal [11], depending on the internal, nuclear composition of the given body. It is thus in general impossible to define a universal Brans-Dicke frame in which the scalar interactions are absorbed in the rescaled metric, for all test bodies.

As a consequence, the equations of motion of test masses, and the response of gravitational antennas, should included in general a direct, non-geodesic coupling of the dilaton charge of the test body to the gradients of the external dilaton field. Such couplings will eventually provide an additional, explicit dilatonic contribution to the equation of geodesic deviation. For string models, this is true even in the so-called "String frame", in which the gravi-dilaton action takes the form of an effective Brans-Dicke action.

Let me start recalling some elementary notion concerning the motion of a test body, described by the Lagrangian $L_{m}$, coupled to a scalar-tensor bakground characterized by the 
Brans-Dicke parameter $\omega$ :

$$
S=\int d^{4} x \sqrt{-g} e^{-\phi}\left[-R+\omega(\nabla \phi)^{2}\right]+\int d^{4} x \sqrt{-g} L_{m}
$$

(metric conventions: +--- ). The variation with respect to the metric and to the dilaton $\phi$ gives, respectively, the field equations (in units $16 \pi G=1$ ):

$$
\begin{aligned}
& G_{\mu \nu}+\nabla_{\mu} \nabla_{\nu} \phi-(\omega+1) \nabla_{\mu} \phi \nabla_{\nu} \phi-g_{\mu \nu} \nabla^{2} \phi+\left(\frac{\omega}{2}+1\right) g_{\mu \nu}(\nabla \phi)^{2}=\frac{1}{2} e^{\phi} T_{\mu \nu}, \\
& R+\omega(\nabla \phi)^{2}-2 \omega \nabla^{2} \phi+e^{\phi} \sigma=0
\end{aligned}
$$

where $G_{\mu \nu}=R_{\mu \nu}-g_{\mu \nu} R / 2$ is the Einstein tensor, and

$$
T_{\mu \nu}=\frac{2}{\sqrt{-g}} \frac{\delta\left(\sqrt{-g} L_{m}\right)}{\delta g^{\mu \nu}}, \quad \sigma=\frac{1}{\sqrt{-g}} \frac{\delta\left(\sqrt{-g} L_{m}\right)}{\delta \phi},
$$

are, respectively, the energy momentum and dilatonic charge density of the test body. For $\sigma \equiv 0$, the combination of the above equations, and the use of the Bianchi and Riemann identities,

$$
\nabla^{\nu} G_{\mu \nu}=0, \quad\left[\nabla^{\alpha} \nabla_{\mu} \phi, \nabla_{\mu} \nabla^{\alpha} \phi\right] \nabla_{\alpha} \phi=R_{\mu}{ }^{\nu} \nabla_{\nu} \phi
$$

leads immediately, for any $\omega$, to the covariant conservation of the stress tensor, $\nabla^{\nu} T_{\mu \nu}=0$, and to the consequent geodesic motion of the test body.

If $\sigma \neq 0$, however, this result is no longer valid. Let me discuss, for simplicity, the physically interesting case $\omega=-1$, corresponding to the lowest-order gravi-dilaton effective action of string theory, in the String frame. By applying the covariant differential operator to the above equations we get

$$
\nabla^{\nu} T_{\mu \nu}+\sigma \nabla_{\mu} \phi=0
$$

which can also be written as

$$
\partial_{\nu}\left(\sqrt{-g} T^{\mu \nu}\right)+\sqrt{-g} \Gamma_{\alpha \nu}^{\mu} T^{\alpha \nu}+\sqrt{-g} \sigma \nabla^{\mu} \phi=0
$$

(for $\omega \neq-1$ there are additional, $\omega$-dependent, dilaton terms).

In order to check explicitly that the motion is not geodesic we can apply the so-called multipole expansion [12], assuming that the gravitational and dilatonic charges of the test body are nonzero only inside a thin "world-tube", centered around the world line $x^{\mu}(\tau)$ of the center of mass. Inside the world-tube, we expand the external fields $\{\Gamma, \nabla \phi\}$ around the position $x^{\mu}$ of the center of mass:

$$
\begin{aligned}
& \Gamma_{\alpha \nu}^{\mu}\left(x^{\prime}\right)=\Gamma_{\alpha \nu}{ }^{\mu}(x)+\left(x^{\prime}-x\right)^{\beta} \partial_{\beta} \Gamma_{\alpha \nu}{ }^{\mu}(x)+\ldots \\
& \nabla^{\mu} \phi\left(x^{\prime}\right)=\nabla^{\mu} \phi(x)+\left(x^{\prime}-x\right)^{\beta} \partial_{\beta} \nabla^{\mu} \phi(x)+\ldots
\end{aligned}
$$


By integrating eq. (7) on the spacelike hypersurface $x^{0}=$ const, and neglecting internal momenta in the point-particle (or pole-particle [12]) approximation, we get

$$
\frac{d}{d x^{0}} \int d^{3} x^{\prime} \sqrt{-g} T^{\mu 0}\left(x^{\prime}\right)+\Gamma_{\alpha \nu}^{\mu}(x) \int d^{3} x^{\prime} \sqrt{-g} T^{\mu \nu}\left(x^{\prime}\right)+\nabla^{\mu} \phi(x) \int d^{3} x^{\prime} \sqrt{-g} \sigma\left(x^{\prime}\right),
$$

where the coordinates $x^{\prime}$ range inside the three-dimensional space-like section of the world tube (the spatial divergence, $\partial_{i}\left(\sqrt{-g} T^{\mu i}\right)$, has been eliminated through the Gauss theorem).

We recall now that, in the point-like limit, the generally covariant stress tensor for a particle of mass $m$, and world-line $x^{\mu}(\tau)$, is given by [13]

$$
T^{\mu \nu}\left(x^{\prime}\right)=\frac{p^{\mu} p^{\nu}}{\sqrt{-g} p^{0}} \delta^{(3)}\left(x^{\prime}-x(\tau)\right)
$$

where $p^{\mu}=m u^{\mu}=m d x^{\mu} / d \tau$. We can define, in the same way, the dilaton charge density in terms of the dimensionless, relative strength $q$ of scalar to tensor forces for the given test body (i.e. the scalar charge per unit of gravitational mass) as:

$$
\sigma\left(x^{\prime}\right)=q \frac{m^{2}}{\sqrt{-g} p^{0}} \delta^{(3)}\left(x^{\prime}-x(\tau)\right)
$$

(the net dilaton charge $q$ may be different for different test bodies, see below). By integrating eq. (9) in the limit in which the radius of the world tube shrinks to zero, $x^{\prime} \rightarrow x$, and multiplying by $m^{-2} p^{0}=m^{-1} d x^{0} / d \tau$, we get finally

$$
\frac{d u^{\mu}}{d \tau}+\Gamma_{\alpha \nu}^{\mu} u^{\alpha} u^{\nu}+q \nabla^{\mu} \phi=0
$$

The motion of the given test body is clearly non-geodesic in a non-trivial gravi-dilaton background, $\nabla \phi \neq 0$ (by the way, this equation implies that the dilaton has to be a shortrange field if $q \gtrsim 1$, to avoid contradictions with tests of the equivalence principle [11, [14]; but I will come back on this point later).

It is now an easy task to compute the dilaton corrections, induced by the charge $q$, to the standard equation of geodesic deviation [13] used to analyze the response of gravitational antennas. We consider two infinitesimally close world-lines, $x^{\mu}(\tau)$ and $x^{\prime \mu}(\tau)$, satisfying eq. (12), and differing by the spacelike separation vector $\eta^{\mu}$, namely $x^{\prime \mu}(\tau)=x^{\mu}(\tau)+\eta^{\mu}(\tau)$. In the equation of motion for $x^{\prime \mu}(\tau)$ we expand the external fields as in (8), and using the motion of $x^{\mu}$ we obtain an expression for the acceleration of the separation vector, $d^{2} \eta^{\mu} / d \tau^{2}$. Shifting to covariant derivatives, $D / D \tau$, the final expression can be written in compact form as

$$
\frac{D^{2} \eta^{\mu}}{D \tau^{2}}+R_{\beta \alpha \nu}^{\mu} u^{\alpha} u^{\beta} \eta^{\beta}+q \eta^{\beta} \nabla_{\beta} \nabla^{\mu} \phi=0
$$


This gives the covariant, relative acceleration of two neighbouring world-lines, for a test body coupled with a charge $q$ to a non-trivial gravi-dilaton background.

We come now to the important point already emphasized at the beginning of this paper. Why the motion of realistic macroscopic test masses, in the gravi-dilaton background of string theory, may be expected to be non-geodesic even in the String frame? In other words, why macroscopic test bodies, in a string theory context, may have composition-dependent dilatonic charges and, consequently, are not adequately described by a pure Brans-Dicke type model of gravity?

To answer this question let me recall that the fundamental fields building up ordinary macroscopic matter, including all loops in the string effective action, are in general coupled non-minimally and non-universally to the dilaton. The weak coupling limit of the dilaton charge $q$ has been carefully estimated in the Einstein frame [0, 11] through a canonical rescaling of fields and masses, and found to depend on two computable (in principle), non-universal loop functions. General arguments then suggest $q \gtrsim 1$ for hadronic matter (typically, $q \sim 44$ for nucleons), while $q \sim 1$ for leptons [11,15]. I will not repeat the computations of [11,7], but I will show here that in the String frame the effective charges are also non-vanishing, and typically of the same order as in the Einstein frame.

To this aim, let me consider a scalar field model matter, $\psi_{i}$, whose gravi-dilaton interactions, including all possible loop corrections, are described by the effective action, in $d+1$ dimensions:

$$
S=\int d^{d+1} x \sqrt{-g}\left[-Z_{R}(\phi) R-Z_{\phi}(\phi)(\nabla \phi)^{2}-V(\phi)+\frac{1}{2} Z_{k}^{i}(\phi)\left(\nabla \psi_{i}\right)^{2}+Z_{m}^{i}(\phi) \psi_{i}^{2}\right] .
$$

Here $Z^{i}$ represent the dilaton coupling function of the field $\psi_{i}$, computed in the String frame, where $g_{\mu \nu}$ is the metric of the conformal sigma-model describing the motion of fundamental strings in the given background. It is always possible, however, to restore the canonical form of all the kinetic terms in the action by introducing a set of rescaled fields $\left\{\tilde{g}_{\mu \nu}, \tilde{\phi}, \tilde{\psi}\right\}$, defined in terms of the old fields and of the coupling functions $Z(\phi)$ (see for instance [7]). The action becomes

$$
S=\int d^{d+1} x \sqrt{-\tilde{g}}\left[-\tilde{R}+\frac{1}{2}\left(\tilde{\nabla} \tilde{\phi}_{i}\right)^{2}-\tilde{V}(\tilde{\phi})+\frac{1}{2}\left(\tilde{\nabla} \tilde{\psi}_{i}\right)^{2}+L\left(\tilde{\phi}, \tilde{\psi}_{i}\right)\right]
$$

where $\tilde{\nabla}$ is the covariant derivative for the Einstein metric $\tilde{g}_{\mu \nu}$, and

$$
L\left(\tilde{\phi}, \tilde{\psi}_{i}\right) \equiv \frac{1}{2} \tilde{\mu}_{i}^{2}(\tilde{\phi}) \tilde{\psi}_{i}^{2}, \quad \tilde{\mu}_{i}^{2}(\tilde{\phi})=Z_{m}^{i}\left[Z_{k}^{i}\right]^{-1}\left[Z_{R}\right]^{2 /(1-d)}
$$

is the canonical matter-dilaton interaction Lagrangian. Its low-energy expansion around the value of $\tilde{\phi}$ which extremizes the dilaton potential (and which can always be assumed to coincide with $\tilde{\phi}=0$, after a trivial shift), 


$$
L\left(\tilde{\phi}, \tilde{\psi}_{i}\right)=\frac{1}{2} m_{i}^{2} \tilde{\psi}_{i}^{2}+\frac{1}{2} \tilde{g}_{i} \tilde{\phi} \tilde{\psi}_{i}^{2}+\ldots
$$

defines the effective masses and dilaton couplings as

$$
\begin{aligned}
& m_{i}^{2}=\left[\tilde{\mu}_{i}^{2}(\tilde{\phi})\right]_{\tilde{\phi}=0}, \\
& \tilde{g}_{i}=\left[\frac{\partial}{\partial \tilde{\phi}} \tilde{\mu}_{i}^{2}(\tilde{\phi})\right]_{\tilde{\phi}=0}=m_{i}^{2}\left[\frac{\partial}{\partial \tilde{\phi}} \ln \tilde{\mu}_{i}^{2}(\tilde{\phi})\right]_{\tilde{\phi}=0} .
\end{aligned}
$$

In the weak coupling limit $Z_{R}=Z_{\phi}=e^{-\phi}$. For $d=3$, in addition, one finds [7] $\phi=\tilde{\phi}$, and the relative coupling strength of scalar to tensor forces (i.e. the dilaton charge in units of the "gravitational charge" $\sqrt{16 \pi G} m_{i}$ ) becomes [11,7], according to eq. (16):

$$
\tilde{q}_{i}=\frac{\tilde{g}_{i}}{m_{i}^{2}}=1+\left[\frac{\partial}{\partial \phi} \ln \left(\frac{Z_{m}^{i}}{Z_{k}^{i}}\right)\right]_{\phi=0} .
$$

In the String frame, on the other hand, the canonical matter field $\widehat{\psi}_{i}=\left[Z_{k}^{i}\right]^{1 / 2} \psi_{i}$ is defined with respect to the unrescaled metric $g_{\mu \nu}$, and the matter-dilaton interaction Lagrangian becomes, at low energy (see eq. (14)):

$$
L\left(\phi, \widehat{\psi}_{i}\right)=\frac{1}{2} \mu_{i}^{2}(\phi) \widehat{\psi}_{i}^{2}, \quad \quad \mu_{i}^{2}=Z_{m}^{i}\left[Z_{k}^{i}\right]^{-1}
$$

In the weak coupling limit, this gives a dimensionless dilaton charge

$$
q_{i}=\left[\frac{\partial}{\partial \phi} \ln \left(\frac{Z_{m}^{i}}{Z_{k}^{i}}\right)\right]_{\phi=0}=\tilde{q}_{i}-1 .
$$

Thus, unless $\tilde{q}_{i}$ is fine-tuned to unity, the dilaton charge is non-universal and non-vanishing both in the String and Einstein frame.

For a macrosopic body the total charge $q$, in the weak field limit, is obtained by summing over all the components, $q=\sum_{i} m_{i} q_{i} / \sum_{i} m_{i}$. Suppose that the body, of $\operatorname{mass} M$, is composed of $B$ baryons with mass and charge $m_{b}, q_{b}$, and $Z$ electrons with mass and charge $m_{e}, q_{e}$. For $Z \sim B, m_{e} \ll m_{b}$, we obtain:

$$
q \simeq B m_{b} q_{b} / M=(B / \mu) q_{b}
$$

where $\mu=M / m_{b}$ is the mass of the body in units of baryonic masses. Since $B / \mu \sim 1$, the total dilaton charge is controlled by the dilaton coupling to baryons, $q_{b}$. If, as suggested in 11],15], $\tilde{q}_{b} \gg 1$, then the dilaton charge of macroscopic bodies is of the same order of magnitude both in the String and Einstein frame, and is composition-dependent (as $B / \mu$ depends on the internal nuclear structure), with variations, across different types of ordinary matter, which are typically of order 


$$
\frac{\Delta q}{q} \simeq \Delta\left(\frac{B}{\mu}\right) \sim 10^{-3} .
$$

Let me come back, finally, to eq. (13), which applies to all models of gravity (not only string theory) in which macroscopic test masses are coupled non-geodesically, with dilatonic charges $q$, to an external scalar-tensor background. Such equation (never considered previously in the gravity-wave literature, to the best of my knowledge), should be taken as the starting point for a general analysis of the response of a detector to scalar oscillations. A detailed computation of the sensitivity to dilatonic waves, based on such equation, is outside the purpose of this paper, and is demanded to further studies and to the work of research group with experience in the analysis of gravitational antennas. However, let me attempt here a naive, qualitative estimate, just to have a first indication of the possible differences induced by the direct coupling to the dilatonic charge of the antenna.

I will follow the standard analysis presented in [16]. For small, non-relativistic oscillations of two test masses, with rest separation $L^{\mu}$, we can put $\eta^{\mu}=L^{\mu}+\xi^{\mu}$, and from eq. (13) we obtain, to first order,

$$
\ddot{\xi}^{i}+R_{k o o}^{i} L^{k}+q L^{k} \partial_{k} \partial^{i} \phi=0
$$

We include restoring and damping mechanical forces, corresponding to a proper oscillation frequency $\omega_{0}$, and to a friction coefficient $\gamma$ :

$$
\ddot{\xi}^{i}+\gamma \dot{\xi}^{i}+\omega_{o}^{2} \xi^{i}+R_{k o o}{ }^{i} L^{k}+q L^{k} \partial_{k} \partial^{i} \phi=0
$$

Consider the response to a monocromatic scalar wave $\phi=\phi_{0} \exp (i k x-i \omega t)$, propagating along the oscillator direction. As we are interested in the direct coupling to the dilatonic charge, we neglect here the gravitational coupling to the metric, and we get the steady-state solution

$$
\xi(t)=\frac{q L k^{2}}{\omega^{2}-\omega_{0}^{2}+i \gamma \omega} \phi_{0} e^{-i \omega t}
$$

(the spatial dependence of the wave has been neglected, assuming $|k x| \ll 1$ throughout the oscillator). For a detector with two masses $M$, vibrating with the above amplitude, we can define a vibration energy $M \dot{\xi}^{2}$, and a corresponding cross section $\sigma$ (the energy dissipation rate per unit of incoming flux) as [16]

$$
\sigma_{\phi}=\frac{M \dot{\xi}^{2} \gamma}{\omega^{2} \phi_{0}^{2}}
$$

Up to this point, the response of the detector to scalar radiation is similar to the case of tensor radiation, with two important differences, however: the additional presence of the 
dilaton charge, which multiplies the incident amplitude, and the fact that the time derivatives of the metric oscillations, $\ddot{h}$ (contained in the Riemann tensor), are replaced by the spatial gradients of the scalar wave, $\nabla^{2} \phi$ (coupled to the dilaton charge). As a consequence, the response (26) of the detector turns out to be proportional to $k^{2}$ instead of $\omega^{2}$. We have now two possibilities.

1) The scalar waves are massless. In this case $k=\omega$, and the reponse is greatly enhanced for waves of resonant frequency $\omega=\omega_{0}$. The cross section, at the resonance,

$$
\sigma_{\phi}=\frac{M q^{2} L^{2} \omega_{0}^{2}}{\gamma}
$$

is the same as the graviton cross section, multiplied however by the dilaton charge $q^{2}$. For the string theory dilaton, unfortunately, the charge in this case gives a very strong suppression factor, because a massless dilaton is incompatible with the present tests of the equivalence principle unless some form of universality is assumed in the string loop corrections [17], thus evading the conclusion of [1], and leading to a very small dilatonic coupling to matter, $q^{2} \ll 1$. This suppression should be valid for any long-range scalar field with compositiondependent couplings. Assuming that the detectors are sensitive enough, however, it should be possible, in this case, to discriminate scalar from tensor signals by comparing the response of different antennas. If, on the contrary, the long range scalar field is universally coupled to matter, then a Brans-Dicke model of gravity is appropriate, and the analysis performed in [1] - [5] can be applied.

2) The scalar waves are massive. In this case the response of the detector is modeldependent. For the string theory dilaton, in particular, the coupling may be large, $q>1$, but then the dilaton mass has to be large enough [11,14,7], i.e. $m \gtrsim 10^{-4} \mathrm{eV} \sim 10^{2} \mathrm{GHz}$, to keep the dilaton corrections to macroscopic gravity below the threshold of present experimental observations 18]. Since $\omega>m$, it seems impossible in this case to match the resonance condition of present gravity wave detectors, with $\omega_{0} \sim 10^{2}-10^{3} \mathrm{~Hz}$. We have always $\omega \gg \omega_{0}, \omega \gg \gamma$, and the cross section becomes

$$
\sigma_{\phi}=M q^{2} L^{2} \gamma\left(\frac{k}{\omega}\right)^{4}
$$

With respect to the resonant graviton cross section, $\sigma_{h}=M L^{2} \omega_{0}^{2} / \gamma$, the response of the detector to massive dilatons, of momentum $k$ and energy $\omega$, is thus suppressed by the ratio

$$
\frac{\sigma_{\phi}}{\sigma_{h}}=\frac{q^{2}}{Q^{2}}\left(\frac{k}{\omega}\right)^{4} \ll 1,
$$

where the factor $Q=\omega_{0} / \gamma$ is in general very large for resonant detectors [16]. 
It should be noted, as a final remark, that in a scalar-tensor model of gravity the oscillations of the scalar background induce oscillations in the scalar sector of the fluctations of the metric. The scalar oscillations of the metric contribute to the total Riemann tensor, and are thus gravitationally coupled to the detector, through the standard equation of geodesic deviation. This is the effect considered in previous papers [1] - [5], which is contained in the

Riemann part of eq. (25), and which is to be added to eq. (26) when computing the full response of the detector.

In some models, the direct coupling to the dilaton charge of the antenna could represent the dominant factor, controlling the response to scalar radiation. Even in that case, however, the results about the possible detection of (massive or massless) dilatons predicted in a string theory context seem to remain pessimistic, in agreement with the conclusions of [5]. Nevertheless, it may be important to note that for dilatons, unlike for gravitons, the direct coupling of the scalar wave to the dilatonic charge of the antenna induces a response proportional to the momentum squared (instead of the energy squared) of the incident particles. Can this help for the experimental detection of massive scalar waves?

\section{ACKNOWLEDGMENTS}

I wish to thank Maura Brunetti, Michele Maggiore and Gabriele Veneziano for useful discussions and comments. 


\section{REFERENCES}

[1] M. Bianchi, E. Coccia, C. Colacino, V. Fafone and F. Fucito, Class. Quantum Grav. 13 (1996) 2865.

[2] M. Bianchi, M. Brunetti, E. Coccia, F. Fucito and J. A. Lobo, Phys. Rev. D 57 (1998) 4525 .

[3] M. Brunetti E. Coccia, V. Fafone and F. Fucito,, Phys. Rev. D 59 (1999) 044027.

[4] M. Shibata, K. Nakao and T. Nakamura, Phys. Rev. D 50 (1994) 7304.

[5] M. Maggiore and A. Nicolis, Detection strategies for scalar gravitational waves with interferometers and resonant spheres, gr-qc/9907055.

[6] T. Harada, T. Chiba, K. Nakao and T. Nakamura, Phys. Rev. D 55 (1997) 2024.

[7] M. Gasperini and G. Veneziano, Phys. Rev. D 50 (1994) 2519.

[8] M. Gasperini, Phys. Lett. B 327 (1994) 314.

[9] M. Gasperini, Relic dilatons in string cosmology, in Proc. of the 12th It. Conference on "General Relativity and Gravitational Physics" (Rome, September 1996), edited by M. Bassan et al. (World Scientific, Singapore, 1997), p. 181.

[10] See for instance M. B. Green, J. Schwartz and E. Witten, Superstring theory, (Cambridge U. Press, Cambridge, Ma, 1987).

[11] T. Taylor and G. Veneziano, Phys. Lett. B 213 (1988) 450.

[12] A. Papapetrou, Proc. Roy. Soc. A 209 (1951) 248.

[13] S. Weinberg, Gravitation and cosmology (Wiley, New York, 1972).

[14] J. Ellis, S. Kalara, K. A. Olive and C. Wetterich, Phys. Lett. B 228 (1989) 264.

[15] See also I. Antoniadis, On possible modifications of gravitation in the (sub)millimeter range, in Proc. of the XXXIV Rencontres de Moriond on "Gravitational Waves and Experimental Gravity" (Les Arcs, January 1999).

[16] C. Misner, K. Thorne and J. A. Wheeler, Gravitation (Freeman and Company, San Francisco, 1973).

[17] T. Damour and A. M. Polyakov, Nucl. Phys. B 423 (1994) 532.

[18] See for instance E. Fischbach and C. Talmadge, Nature 356 (1992) 207. 